beobachteten Fälle von geschlossenen periproktitischen Abscessen, die mit Oxyuren reichlich gefüllt waren. Der erste ist bereits von WEIGMANN veröffentlicht (Berl. klin. Wochenschr. Nr. 27, S. 732. I92I).

Fall IV. 6 jähriger Knabe, der seit Jahren an Würmern leidet, mit heftigem Jucken am Anus, bekommt vor 3-4 Tagen Schmerzen beim Stuhlgang, Schwellung und Rötung am After, dazu Fieber und Mattigkeit. Man findet am Anus und in seiner nächsten Umgebung einige Oxyuren. Rechts und links beiderseits etwa $2 \mathrm{~cm}$ vom Anus entfernt je ein periproktitischer Absceß prail fluktuierend, der bei genauester Untersuchung keinerlei Verbindung mit Rectalschleimhaut und äußerer Haut zeigt. Bei der Incision entleerten sich außer dünnflüssigem Eiter eine Menge lebender Oxyuren. Auch nach der Incision läßt sich eine Verbindung zwischen Darm und Absceßhöhle nicht feststellen, trotz genauesten Suchens. Es wurden nur Oxyurenweibchen gefunden.

Es ist, wie WEIGMANN ausführt, im höchsten Grade wahrscheinlich, daß die im Rectum befindlichen Weibchen, die Darmwand durchwandernd, Infektionserreger mitgenommen und so den geschlossenen periproktitischen $A b s c e B$ verursacht haben. Eine Fistel bestand weder vor noch bei der Operation - erst 4 Tage vorher traten zum ersten Male Schmerzen auf. Noch sicherer scheint uns für diese Annahme der von WEIGMANN genau wiedergegebene Fall von VUILIEMIN ${ }^{1}$ ) $\mathrm{zu}$ sprechen, wo bei einem II jährigen Knaben $3 \mathrm{~cm}$ vom Anus entfernt ein geschlossener periproktitischer Absceß mit Oxyurenweibchen auftrat, der durch eine $2 \mathrm{~cm}$ dicke Schicht vom Rectum getrennt war. Ein neuerlich bei uns beobachteter weiterer Fall spricht ebenfalls für eine die Durchwanderung der Oxyuren durch die Darmwand begleitende Infektion. In den 3 Fällen wurden wunderbarerweise stets nur Oxyurenweibchen gefunden. Das erklärt sich am besten dadurch, daß man annimmt, diese haben sich in größerer Anzahl durch die Darmwand durchgebohrt, wonach der Absceß entstand. Die Männchen bleiben bekanntlich oben im Darm, nur die befruchteten Weibchen gelangen ins Rectum. Die Annahme, daß nur ein oder einige Weibchen durch die Darmwand durchgewandert seien und im $A b s c e B$ durch Eierablegen zu einer Vermehrung der Würmer geführt hätten, ist ebenso wie die, daß Eier im pararectalen Gewebe abgesetzt werden, unwahrscheinlich, weil eben nur Weibchen gefunden wurden. Es sei denn, man dürfte annehmen, daß Männchen nur in den oberen Darmteilen leben können.

Nach den angeführten 5 Fällen, die alle binnen Jahresfrist zur Beobachtung kamen, empfiehlt es sich doch auch wohl für den Chirurgen, den Nematoden erhöhte Aufmerksamkeit zu schenken - sie kreuzen mit ihren krankheiterregenden Wirkungen gar nicht so ganz selten das chirurgische Arbeitsgebiet*).

Literatur: 1) Virchows Archiv 234, I92I. - ${ }^{2}$ ) Aschoffs Lehrbuch Bd. I, I9I3, S. 266. $-{ }^{3}$ ) Zieglers Beiträge 57, 1914. $-{ }^{4}$ ) Zit. nach Christophersen. - 5 ) AIME GuINARD, Affections chirurg. de l'abdomen, Paris igio, 377, S. 326. - 6) W. WolpF, Mediz. Klinik 1917, Nr. 36. - 7) Zentralbl. f. Chirurg., S. i i 77. $\left.{ }_{8}\right)$ Bruns Beiträge 7I, 843. Anm. - ${ }^{9}$ ) Zentralbl. f. Chirurg. I913, S. I I79. - 10) Zentralbi. f. Bakteriol. 74, H. 7. - ${ }^{11}$ ) Frankf. Zeitschr. f. Pathol., I3, H. 3. - 12) Zentralbl. f. Bakteriol. 47. H. 4. 13) Dtsch, med. Wochenschr. I907, Nr. 43. - ${ }^{14}$ ) Zentralbl. f. Bakteriol. 32,358 , r9o2.

*) Anmerkung bei der Korrektur. In der Dtsch. med. Wochenschr. vom 22. IX. I922 berichtet STAHR über 2 Fälle von Darmgeschwïlsten infolge Trichocephaluseinbohrung im Coecum. Bei einem derselben bestand ebenfalls eine Invagination. Die mikroskopischen Befunde sind die gleichen.

Einen weiteren Fall operierten wir vor einigen Tagen bei einer 32 jähr. Frau: Es fand sich eine hühnereigroße Geschwulst im Coecum mit eingebohrten Trichoce. phalen. Dabei phlegmonöse Entzündung der Schleimhaut. Keine Invagination.

Nach abgeschlossener Korrektur haben wir einen weiteren Fall von Tumorbildung im Coecum infolge von Trichocephaluseinbohrung operiert. Eine $3^{8}$ jährige Frau mit einem hühnereigroßen Tumor im Coecalsack, welcher die Appendix verschließt.

\section{ÜBER DIE BEDEUTUNG DER IONENACIDITÄT DES HARNS FÜR ALLGEMEINE KLINISCHE VORGÄNGE.}

\section{Von}

Prof. W. H. VEIL.

Aus der I. medizinischen Universitätsklinik München. (Ditektor: Prof. Dr. v. ROMBERG.)

Unter den verschiedenen Eigenschaften des Harns ist bisher praktisch fast ungenutzt seine Reaktion geblieben. Wohl wurde und wird sie zur Beurteilung lokaler Prozesse in der Blase herangezogen und ihr Verhalten kann bekanntlich differentialdiagnostisch für die Art der - infektiösen - Noxen bedeutungsvoll werden (Coli, Tbc., Go.). Aber der Rückschlu $\beta$ auf ein allgemeineres Geschehen im Organismus blieb bisher aus, trotzdem man seit alters wei $\beta$, da $B$ hier Beziehungen vorhanden sind, die über lokale Nieren- und Blasenveränderung weit hinausgeht.

Die einzige praktische Konsequenz, die man aus der Reaktion des Harns in allgemeiner Beziehung zu ziehen vorschlug, war einmal ihre Beachtung zur Verhütung von Steinerkrankungen besonders bei abnormer Säuerung des Urins (NEUbauer, ERICH MEXER $^{1}$ ) u. a.), sodann auf Grund bekannt gewordener Abhängigkeit der Harnreaktion von der Nagenreaktion zur Beurteilung der letzteren, wenn aus äußeren Gründen das Ausheberungsverfahren versage. Es sei hier besonders auf die Versuchsreihen A. ADLERs ${ }^{2}$, die dieser auf Veranlassung ADAMS LoEBs anstellte, hingewiesen.

Der Grund der Vernachlässigung der Reaktion des Harns ist ein ersichtlicher: Die Schwierigkeiten ihrer brauchbaren Bestimmung waren $z u$ beträchtlich.

Trotz der Bemühungen NAEGELIS ${ }^{3}$ ) und SAHLIS ${ }^{4}$ ), von Moritz $z^{5}$ ) und voN Folis ${ }^{6}$ ) gelang es eigentlich nicht recht, der Methode hrer Bestimmbarkeit Leben zu verleihen. Das Interesse entsprach auf Grund der damaligen, in der Klinik aktuellen biochemischen Anschauungen nicht dem Aufwand an Zeit und Mühe, die die Bestimmung erforderte.

Zunächst war es in erster Linie HoEBER ${ }^{7}$ ), der die neugewonnenen Vorstellungen über das Wesen von Säuren als Ausdruck der Konzentration der freien $\mathrm{H}$-Ionen der Klinik nahelegte. Durch JaNkowsKy ließ er elektrometrische Bestimmungen mittels der Nernstschen Konzentrationsketten anstellen, daneben die Leitfähigkeit der Urine und ihre titrierbare Acidität bestimmen, und legte zum ersten Male in auch dem Nichtphysikochemiker einleuchtender Form die zweierlei Bedeutung der Acidität des Harns dar. Man versteht darunter entweder die Menge von dissoziiertem und undissoziiertem Wasserstoff pro Liter, die an Hydroxyl gebunden werden muß, damit der Harn neutral wird, oder aber nur die Konzentration des dissoziierten Wasserstoffs. Die beiden Aciditäten, die „Titrationsacidität" und die ",Ionenacidität" sind voneinander unabhängige Größen, so daß von den 2 Methoden zu ihrer Bestimmung jede ihre selbständige Bedeutung haben müBte. Eine Reihe von Versuchen über die Verhältnisse am Fieberharn, bei verschiedenen Krankheiten, an hungernden Tieren, zeigte, da $B$ in der Tat die beiden Reaktionen nicht direkt $z u$ vergleichende Größen sind.

Das komplexe physikalisch-chemische System, wie wir es im Blut, in den Körpersäften und im Urin vor uns haben, bringt es mit sich, daß wir für biologische Reaktionen im Organismus nicht mit der Titrationsacidität rechnen dürfen. Hier sind die jeweils augenblicklichen Reaktionsverhältnisse, d. h. die aktuellen Aciditätsgrade von Bedeutung, nicht aber so sehr die ,potentiellen“, wie man sie unter Vorbehalt gewisser Einschränkungen nach OstwaLd nennen kann.

Wollen wir deshalb über Beziehung der Harnreaktion za Geschehnissen im Organismus irgendweicher Art etwas erfahren, wollen wir aus der Harnreaktion Schlüsse auf Vorgänge im Organismus ziehen, so wählen wir zweckmäßigerweise hierzu die Bestimmung der Ionenacidität. Die weitergehenden chemischen Verhältnisse werden dann besser isoliert geprüft, nicht nach einer komplexen Realtion, wie eben der Titrationsacidität, sondern die Alkalien, Säuren, Neutralsalze usw. für sich.

1) 28. Kongre $B$ für innere Med, S, I60 u. I62. IgII.

2) Zentralbl, für innere Med, I9ı9, Nr. 27.

3) Zeitschr, für physiol Chem:e, 30, S. 313. 1000.

4) Lehrbuch der klin. Untersuchungsmethoden rgzo. 2 .

5) Archiv für klin. Med., 80, Heft 5 und 6 .

6) Americ. Journal of Physiol. I, 7. 1903, Nr. V und I905 Nt, T, S, 102

7) HOFMEISTERs Beitr., 3, S. 525. 
Angeregt durch die Beobachtung, die $O$. Neubauer und mein verehrter Lehrer ERICH MEYER $^{1}$ ) auf dem 28. KongreB für innere Medizin zur Diskussion mitteilten, pflege ich n jedem Krankenfall, den ich eingehend untersuche, den Urin außer nach den üblichen Proben auch auf seine Reaktion $z u$ untersuchen, und zwar nicht nur mittels Lackmus, sondern mittels des von $O$. Neubauer zu diesem Zweck angegebenen Indicators, der eine abnorm hohe Ionenacidität qualitativ vorzüglich darstellt, der ätherischen Lackmoidtinktur. Dieser Untersuchungsmethode und überhaupt schon der Achtsamkeit auf die Reaktion des Fiarnes verdanke ich eine ganze Anzahl von Beobachtungen, die auf Beziehungen der Harnreaktion mehr allgemeiner Natur hinweisen; Beziehung vor allem zu vegetativen Neurosen und nervösen Besonderheiten ${ }^{2}$ ). Von ihnen soll hier nicht die. Rede sein. Es kam vielmehr zunächst darauf an, eine physiologische Basis für diese Beziehungen $\mathrm{zu}$ schaffen und nach den Abhängigkeiten der Ionenacidität des Harnes zu forschen ${ }^{3}$ ).

Wir waren genötigt, die Beobachtungen von Grund auf neu anzustellen, auch wo es sich nicht um ganz unerforschtes Gebiet handelte; letzteres gilt von den Beobachtungen über die Kostformen und überhaupt über den Einfluß der Nahrung, wo $\mathrm{HASSELBALCH}^{4}$ ) schon vor Jahren vorangegangen war. Nach unseren Beobachtungen ist gerade die Auffassung HASSELBALCHS von der posdigestiven Aciditätsschwankung des Harns revisionsbedürftig. Ein weiterer Grund für die scheinbare Wiederholung schon bekannter Beobachtungen war der, daß die Darstellung des Einflusses der Kostformen auf die $+\mathrm{H}$ Ionenkonzentration der Körpersäfte unerläßlich zum Verständnis der Gesamtheit des physiologischen Geschehens ist.

Von vornherein ergab sich bei unseren Untersuchungen, daß die Betrachtung der Harnacidität allein ein wesenloser Begriff wäre. Schon meine langjährigen klinischen Beobachtungen nach dieser Richtung hatten das Bedürfnis hervortreten lassen, die Beziehungen zu den Momenten herzustellen, die als Vergleichspunkte herangezogen werden müssen. Naturgemäß konnte es sich in erster Linie nur um die Beziehungen zum Blute handeln. Aus äußeren Gründen wurde nun aber nicht so, wie wir es gerne gewollt hätten, der auf Grund der WINTERSTEINschen Neutralitätstheorie notwendigen $+\mathrm{H}$ Ionenkonzentration des Blutes im allgemeinen bzw. der $\mathrm{CO}_{2}$-Bindungsfähigkeit des Blutes, die von BARcrofr und H. STRAUB ${ }^{5}$ ) als einziger eigentlicher Maßstab für einen Rückschluß auf die biologisch maßgebende Zusammensetzung der $+\mathrm{H}$-Ionenkonzentrationskomponenten verwendbar ist, Beachtung geschenkt. Wir mußten uns mit der Bestimmung der $\mathrm{CO}_{2}$-Spannung im arteriellen Blut begnügen, wie sie durch Analyse der $\mathrm{CO}_{\mathrm{a}}$-Spannung in der Alveolarluft nach HALDANE ${ }^{6}$ ) möglich ist.

Dies war die eine Seite unserer Methodik, die der Erwähnung bedarf, die andere ist die der Bestimmung der Ionenacidität; wir nahmen sie mittels der klinisch sehr zu begrüßenden Indicatorenmethode ohne Puffer von L. MichAELIS ${ }^{7}$ ) vor. Über die Einzelheiten unserer Methodik und auch über ausfübrliche Versuchsanordnungen siehe bei ENDRES ${ }^{8}$.

Wesentlich ist die Art, wie die Versuche anzulegen sind. Es kommt dabei darauf an, daß sämtliche Schwankungen der Ionenacidität zur Darstellung kommen. Der in klinischen Lehrbüchern und Leitfäden der Untersuchungsmethodik aufgestellte Grundsatz, daß die Harnreaktion im gesammelten 24 stündigen Urin festgestellt werden müsse, hat nur ganz extreme Berechtigung. Wenn der 24 stündige Harn beispielsweise für Lackmoidtinktur, deren Umschlag inalkalisch schon bei $p_{\mathrm{B}} 5,4$ bis 5,6 liegt, sauer ist, so bedeutet das ganz extreme Verhältnisse, die allerdings höchstens vielleicht den WahrscheinlichkeitsschluB erlauben, daB alle physiologischen Schwankungen fehlen. Aus der Gesamtzahl einen Rückschlu $\beta$ auf irgendeinen Vorgang zu ziehen, liegt außerhalb der Natur der in

1) Münch, med. Wochenschr. I9r3, Nr. 49 (W. H. VEIL)

3) Herr cand. med. ENDRES widmete sich auf meine Veranlassung dem Studium dieser Frage und stellte die Mehrzahl det Versuche, über die im folgenden berichtet werden soll, mit seiner eigenen Prazon an.

4) Blachem. Zeitschr., 46, S. 403, Igr2.

5) H. STRAUB in ABDERHALDEN's biochem. Arbeitsmethoden, Abt. 4. T, to 0) Journ. of Physiol. I905, T. 32, S. 225.

7) Bochem. Zeitschr. 109, rgzo, S. r65 und Dtsch, med. Wochenschr. 1920 S. I2 und I92I S. 46 und S. 673 .

8) Biochem. Zeitschr. im Erscheinen begriffen.
Frage stehenden Geschehnisse. So erklărt sich, daß unsere Kurven im allgemeinen $1 / 2^{-}$bis 2 stündige Intervalle der sie bestimmenden Punkte aufweisen.

Die Versuche, über die in dieser ersten Mitteilung berichtet werden soll, bestanden darin, daß zunächst der Verlauf der Tageskurve der Ionenacidität durch mehrere Tage hindurch verfolgt wurde.

\section{Tageskurven.}

Kurve I ergibt den Tagesverlauf innerhalb 24 Stunden in einer Form, die eine stets wiederkehrende und demnach die physiologische zu sein scheint. Auf den ersten Blick ist ersichtlich, daß sie in 2 scharf gegeneinander abgesetzte Teile zerfällt, den auf den Tag im engeren Sinne und den auf die Nacht entfallenden Teil. Die Scheidung ist dadurch so scharf, daß der Verlauf der beiden Kurven, derjenige für die Ionenacidität und derjenige für die Kohlensäurespannung, für die Alveolarluft während der Nacht bzw. während der Zeit des Schlafes ein prinzipiell anderer als am Tage ist.

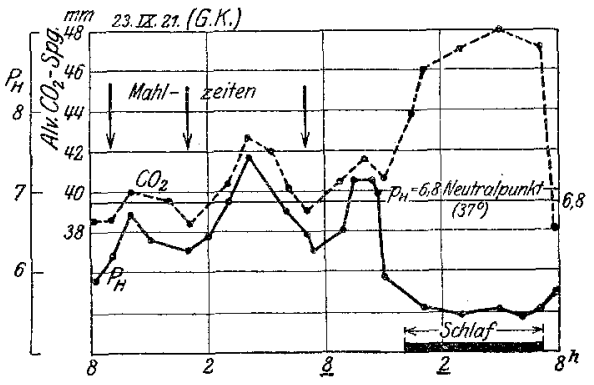

Kurve $\mathrm{I}^{\mathbf{1}}$. Abszisse: Zeit, Ordinate: $p_{\mathrm{H}}$ bzw. $\mathrm{CO}_{2} \mathrm{~mm}$. Tageskurve der alv. $\mathrm{CO}_{4}$ Spannung und des $p_{\Pi}$ des Harns. $p_{\mathrm{H}^{-}}$Kurve ausgezogen; $\mathrm{CO}_{2}$-Kurve punktiert; Pfeile geben die eingenommenen Mahlzeiten an.

Der Tagesverlauf ist der folgende: Einhaltung einer Durchschnittslinie für $p_{\text {日 }}$ zwischen 6,2 und 7,2 , für die $\mathrm{CO}_{2}$ Spannung $z$ wischen $3^{8}$ und 43 .

Der Nachtverlauf dagegen weist eine gleichmäßige niedere Ionenacidität im Urin zwischen 5,2 und 5,75 auf, Werte also, die nahezu auf einer Linie liegen. Die $\mathrm{CO}_{2}$-Spannung bewegt sich in extrem hohen Werten, und zwar zwischen 44 und $48 \mathrm{~mm} \mathrm{Hg}$.

Die Abweichung der mittleren Linie im Tagesverlauf beider Kurvenlinien sind, wie sofort ersichtlich ist, durch postdigestive Schwankungen bedingt. Mit Regelmäßigkeit folgt jeder Mahlzeit eine derartige Zacke, und zwar ist sie um so höher, je größer die Mahlzeit ist. Die höchste Zacke folgt der Mittagsmahlzeit. Nach $2-3$ Stunden erreicht sie ihren Höhepunkt. Bei geringer Nahrungszufuhr wird das Maximum früher erreicht als bei reichlichem Essen. Während der nächsten $2-3$ Stunden fallen dann die Kurven ab.

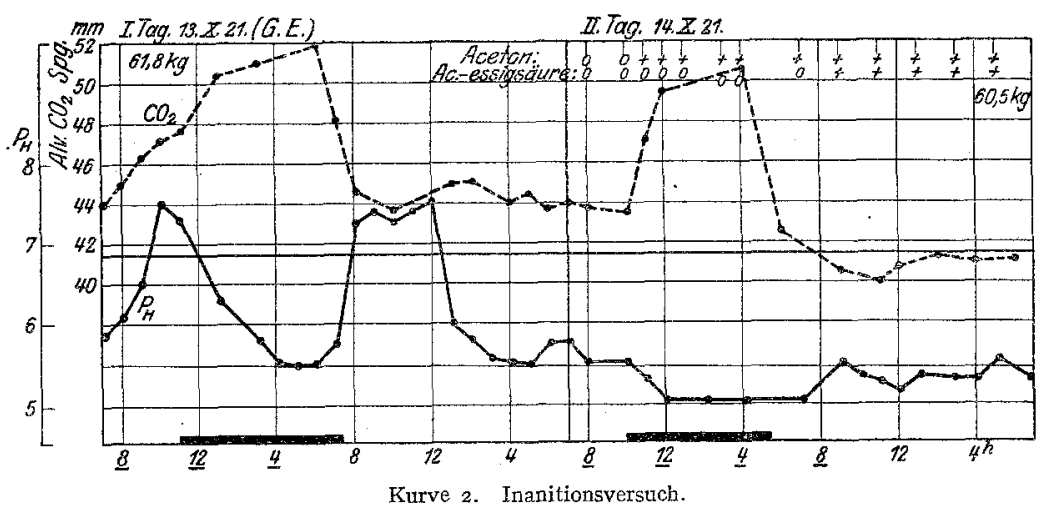

a) Abhängigkeit der Kurven von der Nahrungsaufnahme und Koslform. Bedeutung der Salzsäureabscheidung im Magen.

Die Abhängigkeit der Schwankungen in den Kurvenlinier von den Mahlzeiten ist eine frappante. Immerhin ist ler Beweis dafür, $\mathrm{da} ß \widehat{B}$ diese Abhängigkeit keine zufällige ist, $z \mathfrak{u}$ erbringen.

Den Beweis demonstriert die Kurve 2.

Sie stellt einen Inanitionsversuch von derselben Versuchsperson dar, von der Kurve I aufgenommen ist. Hier flachen sich vom I. Hungertag an die Linien beider Kurven während des Tages-

1) Ább. I ist eine typisch Kurva au; dar Schar vo: übar 50 an 8 verschied nen Personen beobachteten Tagesschwankungsn. 
verlaufs ab. Nur die Nachitschwankungen bleiben bestehen. In der Ionenacidität ist in den frühen Morgenstunden des I. Hungertags eine alkalische Zacke ohne jegliche Nahrungszufuhr vorhanden, die sonst fehlt. Auf 'sie wird noch zurückzukommen sein. Sie ist der Protoyp der sog. spontanen Alkaliurie.

Die Mahlzeiten als solche bewirken eine Veränderung der Ionenacidität des Harns, und zwar eine Abnahme des Säuregrades unter Näherung oder sogar Uberschreitung des Neutralpunktes $\left(p_{H} 6,8\right)$ sowie eine Zunahme der Kohlensäurespannung der Alveolarluft. Es bliebe runmehr zu diskutieren übrig,

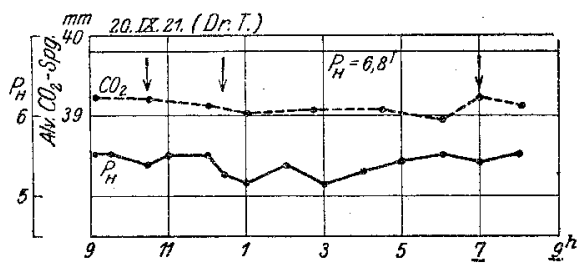

Kurve 3. Nahrungsaufnahme beim Anaciden.

welches Moment an der Nahrungszufuhr es ist, wodurch diese Schwankung verursacht wird. In Betracht kommen zwei Möglichkeiten, der Einfluß der Nahrungszufuhr auf den Stoffwechsel und die Abscheidung der Salzsäure in den Magen. Beides hydrate in seiner Nahrung zugeführt erhielt (Kurve 3). Bei ihm hat die Mahlzeit nicht den geringsten Einfluß auf die Ionenacidität des Harns und die Kohlensäurespannung der Alveolarluft ${ }^{1}$.

Ebenso klar liegen aber auch die Verhältnisse für die Kostformen. Allerdings ist die Höhe der Ausschläge, die die Linien der Ionenacidität und $\mathrm{CO}_{2}$-Spannung geben, durchaus abhängig von der Art der eingeführten Nahrung.

Reine Fleischdiät (die Versuchsperson, von der die Kurve 4 stammt, genoß ausschließlich amerikanisches Büchsenfleisch, Cornedbeef oder frisches Rind- und Kalbfleisch, daneben nur noch Leitungswasser).

Reine Kohlenhydratkost hingegen, die bei unserer Versuchsperson ausschließlich in Semmelnahrung bestand, schuf am Ende der 6 Kohlenhydratversuche Verhältnisse, wie sie Kurve 5 zeigt.

Stark erhöhter Tagesdurchschnitt beider Linien ist charakteristisch. Die postdigestiven Erhebungen der Linien sind weniger spitz, mehr plateauartig. Während bei Fleischkost die Erhebung meist nach 2 Stunden ihren Höhepunkt er-reicht, weist Kohlenhydratkost dieselbe erst nach 3-4 Stunden auf.

Utber den Einfluß der Ernährung läßt sich also sagen, daß die Schwankungen der Ionenacidität des Harns und der $\mathrm{CO}_{2}$ Spannung mit der Salzsäuresekretion des Magens und nicht
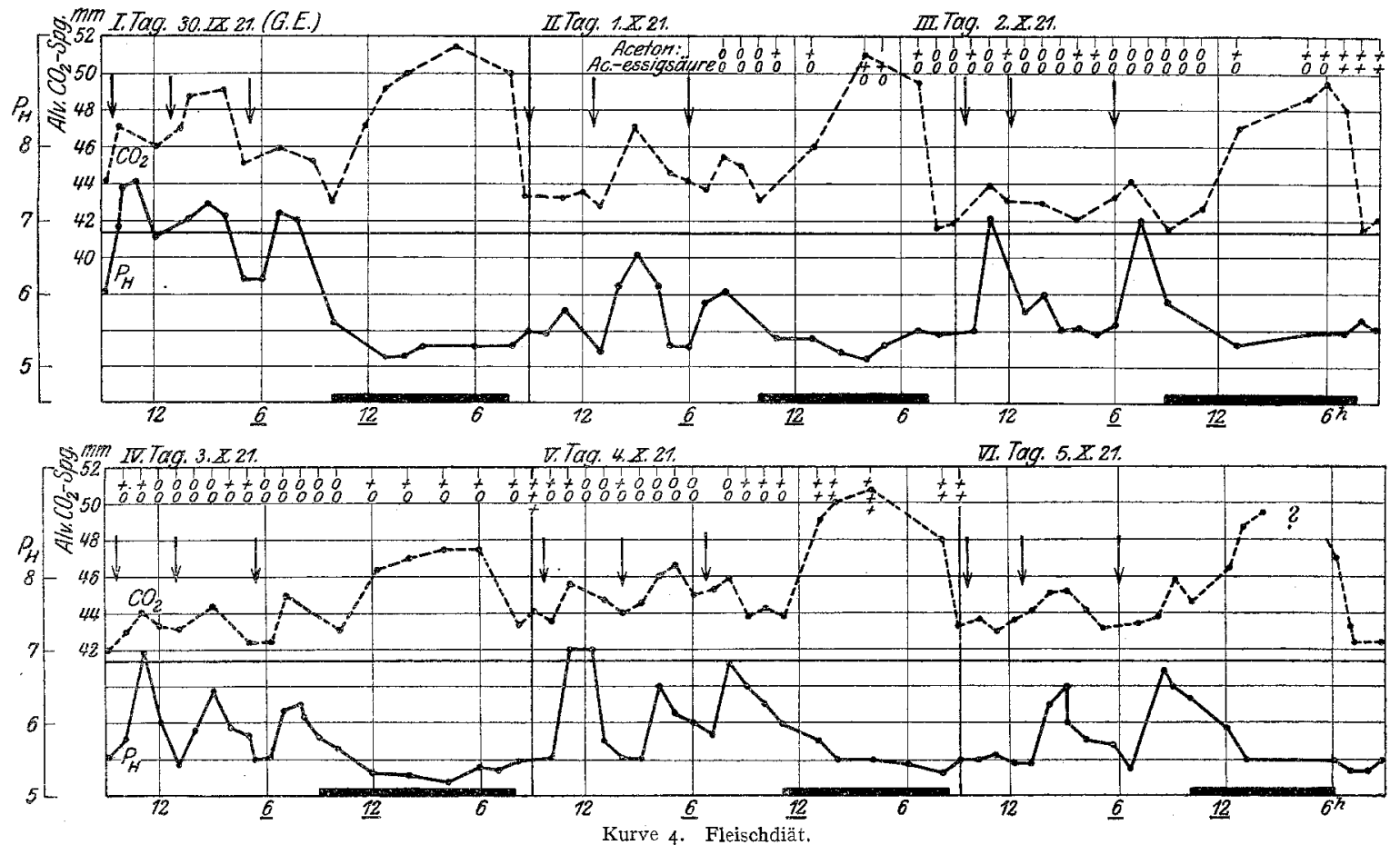

wird auch in der Literatur. erwogen, von HASSELBACH ${ }^{1}$ ) dahin beantwortet, daß speziell der Kohlenhydratstoffwechsel für die Tagesschwankungen der Harnreaktion verantwortlich zu machen sei. Nach Hasselbach veranlaßt nur eine Kost, die Kohlenhydrate enthält, die betreffenden Reaktionsänderungen, und zwar um so stärker, je kohlenhydratreicher sie ist. Fleischdiät, die doch noch mehr als die vegetarische die Salzsäuresekretion des Ventrikels befördert, gebe einen sauren Harn fast ohne Tagesschwankung der Reaktion. Die mit der kohlenhydratreichen Mahlzeit eingeführten Elektrolyte bewirlaten also die Säureerniedrigung des Harns; denn am I. Inanitionstag, sogar bis 24 Stunden nach der letzten Mahlzeit habe der Harn einen auffallend hohen $p_{\mathrm{H}}$. Erst am 2. Inanitionstag, nachdem der Giykogenvorrat verbraucht ist, fange der Harn an, die niedrigeren $p_{\mathrm{E}}$-Werte der Fleischfettverbrennung anzunehmen.

Daß der wesentliche Anteil auf die Rechnung der Salzsäureabscheidung im Magen zu setzen ist, beweist die Beobachtung eines Achylikers mit vollständiger Anacidität nach jeglicher Nahrungszufuhr, trotzdem er reichlich Kohlen- mit der Kostform zusammenhängen. In unserem Versuche tagelang durchgeführter reiner Fleischdiät kam es zu starker

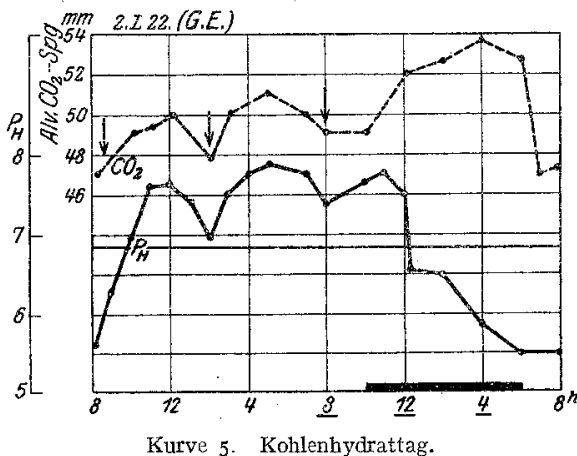

Ketonurie; demnach waren die Glykogenvorräte des Körpers weitgehend erschöpft; trotzdem aber blieben die Kurven-

1) Wäbrend unserer Versuche publizierte PORGES zusammen mit KONDERS einen Fall von Anacidität, der dasselbe Phänomen darbot (Dtsch, med, Wochensehr. Ig2x, H. I5ff.). 
zacken bestehen. Umgekehrt war gemischte, kohlenhydratreiche Kost beim Achyliker nicht von den gewöhnlichen Bewegungen in beiden Kurvenlinien gefolgt; beide Kurven waren nahezu gerade.

Die Salzsäuresekretion des Magens führt zu einem Verlust des Körpers an Alkali und $\mathrm{zu}$ vermehrter $\mathrm{CO}_{2}$-Anhäufung im Blute infolge Abwanderung der $+\mathrm{H}-$ Ionen nach dem Darmtraktus. Man sollte nun annehmen, daß dieser Bewegung eine entgegengesetzte Bewegung der Zeit des Hungerzustandes entspräche. Das ist aber im Laufe des Tages nicht der Fall. Die Hungerlinie liegt naturgemäß niedriger als der Durchschnitt der postdigestiven Zacken liegt (s. Kurve 2); aber dabei ist zu berücksichtigen, daß hier auch die Verbrennungsprozesse auf ihren endogenen Unterhait beschränkt bleiben, und daß die am reichlichsten $\mathrm{CO}_{2}$ liefernden Körper, die Kohlenhydratdepots des Organismus, am frühesten erschöpft sind.

Man mu $B$ also wohl annehmen, daß die im Magen ausgeschiedenen sauren Valenzen in einer Art und Weise zur Resorption gelangen, ohne da $B$ es zu wesentlichen Verschiebungen im Gleichgewicht der Ionen kommt, wohingegen ihre Abscheidung so schlagartig erfolgt, daß das Gleichgewicht in beträchtlicher Weise verändert wird. An und für sich verfügt wohl der Darm fortdauernd über größere Mengen von Alkalien, eine Art von stabiler Reserve, die der Bindung der Säure dient; dieser Umstand würde den Unterschied des Einflusses der Sekretion der Magensäure gegenüber ihrer Resorption erklären.

b) Einfluß des Schlafes. Bedeutung. des Nervensystems.

Der Verlauf der 24 stündigen Tageskurven für $p_{\mathrm{H}^{*}}$ und $\mathrm{CO}_{2}$-Spannung wies die grundlegende Scheidung in Tages- und Nachtabschnitte auf, besser gesagt - bei näherer Beobachtung - in Wach- und Schlafzeiten. Die Scheidung ist auch vorhanden in der Kurve des Inanitionsversuches und, wie ich versichern darf, ohne die diesbezügliche Kurve beizubringen, in der des Achylikers. Sie hat also nichts mit der Nahrungsaufnahme, nichts mit der Salzsäureabscheidung im Magen zu tun.

Mit dem Einsetzen des Schlafes fällt das $p_{\mathrm{H}}$ des Harns ab, die sauren Valenzen im Harn nehmen zu. Nach den bisherigen Beobachtungen der postdigestiven Bewegungen müBte erwartet werden, daB die $\mathrm{CO}_{2}$-Spannung gleichfalls abnimmt; in allen bisherigen aufgeführten Kurven liefen die beiden Linien ja ziemlich genau parallel. Hier aber divergieren sie; die Kohlensäurespannung steigt an. Schon durch H. STraub ${ }^{1}$ ) und durch PORGES ${ }^{2}$ sind wir über diese letztere Tatsache unterrichtet. Auf Grund der Winterstern schen Neutralitätstheorie ist anzunehmen, daß eine Unterempfindlichkeit des Atemzentrums während des Schlafes vorliegt und infolgedessen das $c_{\mathrm{H}}$ des Blutes, das bekanntlich für die Regulation des Atemzentrums maßgebend ist, zunimmt. Daran wirkt in erster Linie und einerseits die Blutkohlensäure, andererseits die weniger ins Gewicht fallende, aber doch auch bedeutungsvolle Phosphorsäure (Verschiebung des Gleichgewichts der Phosphate zugunsten einer Vermehrung der sauren Phosphate) mit; es kommt noch dazu die Wechselwirkung zwischen dem Phosphat- und Carbonatgemisch; die Kohlensäure übt bei einer bestimmten Konzentration Einfluß auf das Dinatriumphosphat aus, so daß auch hier wieder eine Verschiebung nach den sauren Phosphaten erfolgt. Die natürliche Folge ist demnach auch theoretisch eine Zunahme der Ionenacidität des Harns, und dieses wurde in der Tat von uns auch festgestellt.

Normalerweise ist die Menge des Nachtharns gegenüber dem Tagharn vermindert, das spezifische Gewicht ist gesteigert; man spricht von dem höher gestellten Nachtharn. In einer späteren Mitteilung soll auf die Beziehungen dieser Eigentümlichkeiten zu den eben berührten Gesetzmäßigkeiten eingegangen werden. Hier sei nur darauf hingewiesen, daß es sich auch hierbei nicht um Zufälligkeiten handelt.

\section{e) Spontane Alkaliurie.}

In Kurve 2 (Inanitionsversuch) zeigt sich, worauf schon kurz die Redo kam, bald nach dem Erwachen und gleich-

1 u. $\left.{ }^{2}\right)$ I. C.

3) Dtsch. Zeitschr. f. klin. Med. zeitig mit dem Abfall der Kohlensäurespannung, zum Tagesdurchschnitt bezichungsweise Wachwert eine unvermittelte Zunahme des $p_{\mathrm{H}}$, d. h. Alkalescenz des Harns auf ganz beträchtliche Werte (von $p_{\mathrm{H}} 5,6$ bis $p_{\mathrm{H}} 7,75$ ). Diese Bewegung ging spontan vor sich, sie wiederholte sich bei unserer haupt sächlichen Versuchsperson des öfteren, zeigte sich aber auch bei anderen mitunter. Tür ihre Erklärung dürfte an das für die Bewegung während des Schlafes Gesagte anzuknüpfen sein. Die plötzliche Verschiebung der Verhältnisse durch das Wiedererwachen der normalen Erregbarkeit des Atemzentrums mit Beendigung des Schlafzustandes macht sich hier geltend. Meist scheint sich der Ausgleich zwischen Blut und Gewebe abzuspielen. Gelegentlich aber kommt er auch am Urin in derartigen alkaliurischen Zacken zum Ausdruck.

Bekanntermaßen [BECKMANN $\left.{ }^{3}\right)$ ] kann das Atemzentrum auch durch andere nervöse Einflüsse als den des Schlafes in seiner Erregbarkeit umgestimmt werđen; insbesondere können nervöse Reize seine Erregbarkeit ganz plötzlich steigern. In solchen Fällen kann das Bild dasselbe sein, wie in der Schwankung der Kohlensäurespannung und der Ionenacidität des Harns im Anschluß an die Schlafschwankung des ersten Inanitionsversuches (Kurve 2), Abfall der Kohlensäurespannung - in diesem Falle durch Schreck oder irgendwelche anderen psychischen Erregungen. Diese plötzliche Veränderung des Atemzentrums kann sich - aber nur unter Umständen in Alkaliurie äußern. Es handelt sich hier nicht um theoretische Uberlegungen, sondern von uns gemachte tatsächliche Beobachtungen.

\section{Künstliche Einwirkung auf die Ionenacidität.}

\section{a) Abnorme Muskeltätigkeit.}

Jeder über das Maß des täglichen Lebens hinausgehende Muskelanspruch vermag die Ionenacidität des Harns zu steigern (Kurve 6). Dieser Bewegung geht regelmäßig ein explosionsartiges Ansteigen der $\mathrm{CO}_{2}$ voraus. Es ist der Ausdruck der plötzlich vermehrten Verbrennung und Kohlensäurebildung. Zunächst bleibt die Ionenacidität des Harns unverändert (Versuch 2); dann aber mit dem Abfall der Kohlensäurekurve nimmt sie zu. Das Verhalten ist ohne weiteres verständlich, wenn man an den Stoffwechsel des Muskels und die vermehrte Milchsäurebildung denkt. Nach der Regulierung der Kohlensäure spielt sie die entscheidende Rolle auf den Verlauf unserer Kurven. Bekanntermaßen ist der Arbeitsharn „,milchsäurereich".

\section{b) Medikamentöse Einwirkung,}

Unsere wirkungsvollsten Medikamente sind für den Verlauf unserer Kurven von Bedeutung. Hier soll nur paradigmatisch das Wesentliche gezeigt werden. Morphium wirkt nach dem Vorbild des Schlafs. Zunahme dér Ionenacidität, Abnahme der Erregung des Atemzentrums, infolgedessen $\mathrm{Zu}-$ nahme der Kohlensäurespannung.

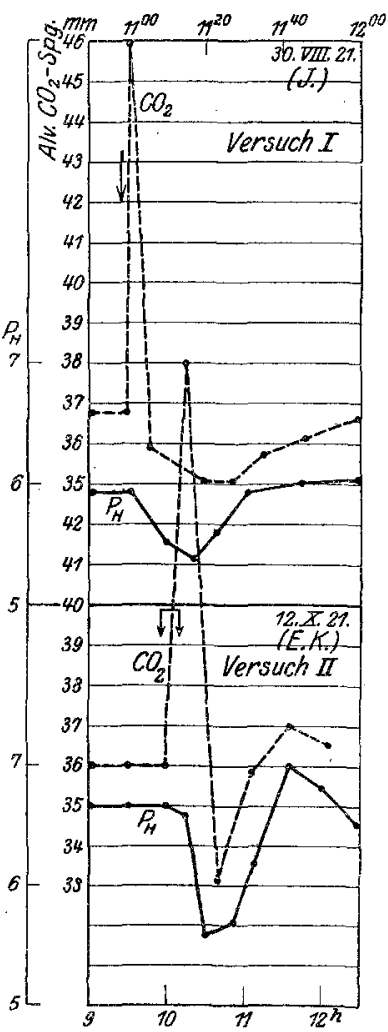

Kurve 6. Muskeltätigkeit.
Die Coffeinwirkung (2. Teil der Kurve 7 ) ist komplizierter, da sie diphasisch verläuft. An der $p_{\mathrm{H}^{-}}$Kurve des Harns drückt sich die Wirkung in einem alkaliurischen Plateau aus, das I2 Stunden lang ziemlich fixiert erscheint. Freilich ist nach 5 Stunden eine vorübergehende Tendenz zum Absinken angedeutet. Mit dem Anstieg der $p_{\mathrm{H}}$-Kurve fällt die Kohlensäurckurve ab ( $5 \mathrm{~mm}$ gegenüber dem Ausgangswert); nach 2 Stunden kehrt sie zum Ausgangswert zurück, und zu dieser Zeit 
tritt eine neuerliche Steigerung der Alkaliurie hervor, die sich nunmehr auf ihrem Plateau noch 7 Stunden lang hält.

Nach der Kohlensäurespannungskurve ist die Wirkung des Coffeins auf das Atemzentrum nach 5 Stunden abgeklungen.

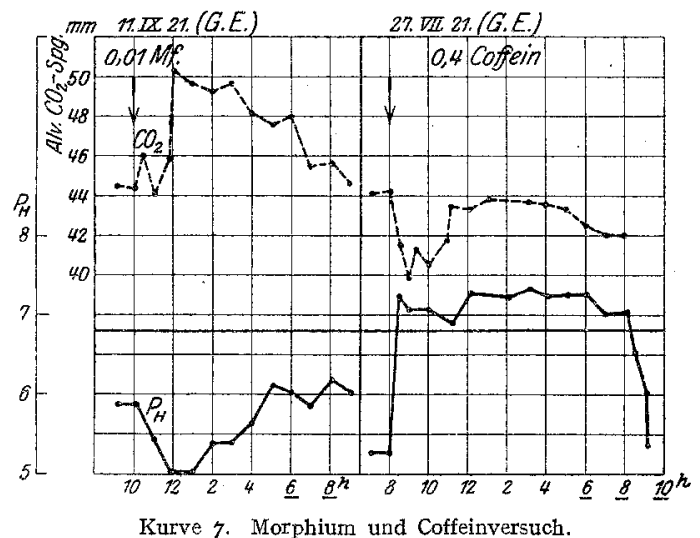

Sie bestand in Steigerung seiner Erregbarkeit und bedingt damit Ausscheidung der freiwerdenden alkalischen Valenzen (siehe unter spontaner Alkaliurie). Mit der Erholung des Atemzentrums aber wird nun die Alkaliurie noch deutlicher, und hier beginnt also eine 2. Phase der Wirkung in die Erscheinung zu treten.

Durch die Versuche Ellingers ist es neuerdings wahrscheinlich gemacht worden, $\mathrm{da} B$ die Wirkungsweise des Coffeins als Diureticum in der Verminderung des Quellungsdruckes der Eiweißsole im Blut zu suchen ist. Der Entquellung der Blutkolloide läuft also eine Alkaliurie parallel, die länger andauert, als die Wirkung des Coffeins auf das Atemzentrum.

Auch die Digitaliswirkung wurde untersucht. Es führt sich die diesbezügliche Beobachtung auf einen zunächst etwas entlegenen Gedankengang zurück. Vorausgeschickt sei, daß gerade die Digitalis sich naturgemäß nicht in gleicher Weise $z u$ den in Frage stehenden Versuchen eignet, wie andere Medikamente, $z$. B. Coffein und Morphium; ihre Wirksamkeit tritt infolge der besonderen Bedingungen der Resorption und der Langsamkeit, mit der sie die Stätte ihrer Wirksamkeit erreicht, zu allmählich hervor; infolgedessen ist immer damit zu rechnen, daß die das ganze Kurvenbild beherrschenden postdigestiven Schwankungen der Ionenacidität und Kohlensäurespannung die eventuell durch das Mittel hervorgerufenen Schwankungen verschleiern.

Als Versuchsobjekt wurde deshalb der oben mitgeteilte Fall von Achylia gastrica mit völliger Anacidität gewähit. Entscheidend für den Versuch war ein therapeutischer Gedanke, und zwar der, daß die Digitalis als Vagusmittel vielleicht eine die Salzsäureabscheidung im Magen befördernde Wirkung haben könnte, eine Annahme, die sich nachher als irrig erwies.

Der Fall eignete sich deshalb aber zu der genannten Beobachtung ganz einzigartig, weil eine I 4tägige Beobachtung ergeben hatte, $\mathrm{da} B$ niemals irgendwelche Schwankungen der Ionenacidität und der Kohlensäurespannung mit Ausnahme der normalen Nachtschwankungen vorkamen; bezüglich der Ionenacidität war letztere auch nur geringfügig. Der Harn war dauernd stark sauer, zeigte sogar das Phänomen des Ausfallens von freier Harnsäure, sofort nach der Miktion.

Frühmorgens wurden $2 \mathrm{ccm}$ Digipurat intravenös gegeben (Kurve 8). Um rie fiel zum ersten Male, seit wir den Fall überhaupt beobachteten die Kohlensäurespannung um $2 \mathrm{~mm}$ und in der Folgezeit noch etwas tiefer ab. Dieser Bewegung entsprach in der Ionenacidität des Harns eine von $\mathrm{I}^{30}$ bis $4^{30}$ sich bemerkbar machende Abnahme der Acidität von $p_{\text {H }}$ 5, o auf $p_{\text {H }} 6,7$. Damit war die Wirkung dann abgeschlossen.

2 Tage später wurde Digipurat innerlich verabreicht, und zwar in Dosis von 3 Tabletten pro die. Nach Ablauf von 36 Stunden machte sich diesmal eine im Vergleich mit der intravenösen Applikation gleichsinnige Bewegung in beiden Kurven geltend, die bis I2 Stunden nach Aufhebung der Medikation anhielt. Sie bestand nicht etwa wie im Coffeinversuch in einem Plateau der Kurven, sondern in unregelmäßigen Zacken, die an die spontane Alkaliurie erinnern.
Auch die Digitalis, und gerade sie also vermag selbständige Wirkungen im Ionengleichgewicht hervorzurufen. Die Wirkungen haben nichts mit der Herzwirkung zu tun, wie in einer zweiten späteren Mitteilung gezeigt werden kann. Thre Erklärung dürfte die der Spontanalkaliurien sein. Nahe

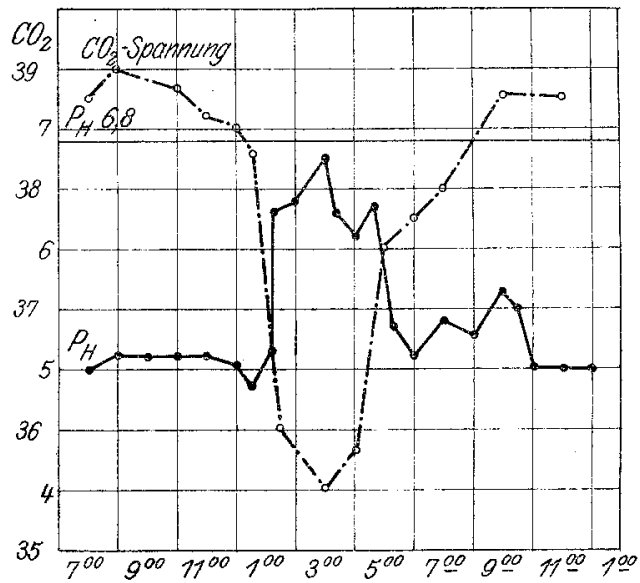

Kurve 8. Digitaliswirkung (intravenöse Infusion von $2 \mathrm{ccm}$ Digipuration Knoll frïh $8^{* 0}$ ) am herzgesunden Achlorhydriker.

Beziehungen scheinen mir vor allem zu der diuretischen Wirkung des Mittels zu bestehen, die auch nicht, wie so oft angenommen wird, Folge der Herzwirkung ist, sondern die einen durchaus selbständigen Charakter hat. Auch am Herznormalen kann im Anschluß an Digitalis, bzw. Strophanthin, Diurese beobachtet werden.

\section{c) Der Aderlaß:}

Es erscheint von Bedeutung, daß die Fluxion aus den Geweben nach dem Blute, wie sie dem Aderlaß folgt, eine Bewegung im Ionengleichgewicht mit sich bringt, erkennbar an einer alkaliurischen Verschiebung der Ionenacidität im Harn und Steigerung der Kohlensäurespannung (Kurve 9). Der der Kurve zugrundeliegende Aderlaß betrug $500 \mathrm{ccm}$ Blut. 2 Stunden später kam die Bewegung in Gang.

In der Hauptsache wird man hier an die Wirkung der ins Blut einströmenden Flüssigkeit

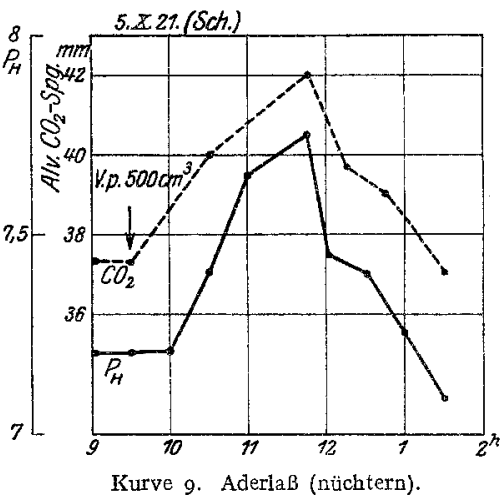
zu denken haben, die vermehrte alkalische Valenzen, darunter reichlich Carbonate, ins Blut bringen. Daneben aber wirkt auch die Verminderung der Blutkörperchen direkt mit. Durch Hasselbach und LundSGaRd wissen wir, daß das Blut bei gegebener Kohlensäurespannung um so saurer reagiert, je mehr es Blutkörperchen besitzt. Ihre Verminderung durch den Aderlaß wirkt also für das Blut an und für sich schon alkalisierend.

Die Summe der im vorstehenden beobachteten Gesetzmäßigkeiten für die Ionenacidität des Harns im Verein mit der Beobachtung der Kohlensäurespannung im arteriellen Blute weist darauf hin, welch feines Reagens wir in dieser Eigenschaft des Harns besitzen und inwieweit wir durch ihn in die Gleichgewichtsverschiebungen der Ionen innerhalb des Organismus hineinsehen können. Seine Beziehungen sind so lange übersehbar, als die Nieren die Fähigkeit der Anpassung an die im Blute vor sich gehenden Verschiebungen besitzen. Wie sich die Verhältnisse unter pathologischen Bedingungen gestalten, darüber soll später eine zweite Mitteilung berichten, 